\title{
Long non-coding RNA FAF1 promotes intervertebral disc degeneration by targeting the Erk signaling pathway
}

\author{
DAGUO MI ${ }^{1}$, CHUNYUE CAI $^{2}$, BIN ZHOU $^{1}$, XUAN LIU $^{3}$, \\ PEIDE MA ${ }^{1}$, SHUIJIE SHEN ${ }^{4}$, WEI LU ${ }^{1}$ and WEI HUANG ${ }^{1}$ \\ ${ }^{1}$ Department of Orthopedics and Traumatic Surgery, Nantong Municipal Hospital of Traditional Chinese Medicine, \\ Nantong, Jiangsu 226001; ${ }^{2}$ Department of Orthopedics and Traumatic Surgery, Qidong Municipal Hospital of \\ Traditional Chinese Medicine, Nantong, Jiangsu 226200; ${ }^{3}$ Department of Orthopedics and Traumatic Surgery, \\ The Second People's Hospital of Binzhou, Binzhou, Shandong 256800; ${ }^{4}$ Department of Oncology, \\ Nantong Municipal Hospital of Traditional Chinese Medicine, Nantong, Jiangsu 226001, P.R. China
}

Received April 12, 2017; Accepted September 13, 2017

DOI: $10.3892 / \mathrm{mmr} .2017 .8237$

\begin{abstract}
Intervertebral disc degeneration (IDD) has become the most common cause of low-back pain, and it imposes a heavy burden on patients with IDD and society. The effects of long non-coding RNAs on the proliferation and development of IDD have attracted increasing attention. The present study aimed to investigate the role and molecular mechanism of Fas-associated protein factor-1 (FAF1) in IDD. The expression of FAF1 was detected by reverse transcription-quantitative polymerase chain reaction. CCK-8 and immunofluorescence staining were used to determine cell proliferation. Flow cytometry was performed to measure the cell cycle and apoptosis. Western blotting was used to test p-Erk expression. The results of the present study demonstrated that the expression of FAF1 was upregulated in patients with disc bulging, herniation and IDD, and the expression of FAF1 was positively correlated with the grade of disc degeneration according to the patients' Pfirrmann score. The overexpression of FAF1 in nucleus pulposus (NP) cells promoted cell proliferation by increasing the percentage of cells in the S-phase of the cell cycle. The expression of phosphorylated extracellular signal-regulated kinase (Erk), a possible target of FAF1, was consistent with the expression of FAF1. In addition, it was elucidated that inactivation of the Erk signaling pathway by PD98059 reversed the effect of FAF1 on NP cell proliferation. Taken together, these results demonstrated that FAF1 was vital in the proliferation
\end{abstract}

Correspondence to: Dr Bin Zhou, Department of Orthopedics and Traumatic Surgery, Nantong Municipal Hospital of Traditional Chinese Medicine, 41 Jian-she Road, Nantong, Jiangsu 226001, P.R. China

E-mail: zhoubin6230@qq.com

Key words: long non-coding RNA, Fas-associated protein factor-1, intervertebral disc degeneration, extracellular signal-regulated pathway of NP cells by modulating the Erk signaling pathway, which suggests that FAF1 may be a novel marker in the early diagnosis of IDD and a therapeutic target for patients.

\section{Introduction}

Intervertebral disc degeneration (IDD) has become the most common cause of low-back pain, which seriously affects the lives of patients and leads to $10 \%$ of patients becoming chronically disabled $(1,2)$. Patients with IDD exhibit characteristics, including the proliferation of intervertebral disc tissues, which consist of the central gelatinous nucleus pulposus (NP) and the outer lamellar annulus fibrosus (3). The degeneration of intervertebral discs is caused by multiple factors, including aging, physical loading, genetic predisposition and lifestyle (4,5). Accumulating pathological conditions result in the abnormal degradation of proteoglycan and type II collagen generated by NP, leading to the remodeling of disc structures and vertebrae (3). Increasing studies have demonstrated that a large number of cellular events are involved in the degeneration of intervertebral discs, ranging from gene expression to protein synthesis. Among these events, the complex progression of gene expression has been found to be vital in IDD (6). Long non-coding RNAs (lncRNAs), as an important factor in gene expression in IDD, have attracted substantial attention in previous years (7-9).

It is known that lncRNAs comprise a variety of transcriptional byproducts of the mammalian genome without protein-coding function (2). They have been identified to be important regulators in differential biological functions, including genome imprinting, gene expression, epigenetic regulation and chromatin modification (10-12). In addition, increasing evidence has shown that the dysregulation of lncRNAs exerts significant function in several human diseases, including ovarian cancer, hepatocellular carcinoma, colorectal cancer and fatty liver disease (13-16). Fas-associated protein factor-1 (FAF1) is an lncRNA and is a member of the Fas death-inducing signaling complex, as its name suggests (17). A previous study indicated that FAF1 is upregulated in IDD (18). However, to the best of our knowledge, the role and molecular 
mechanism underlying the effect of FAF1 in IDD has not to be investigated.

The present study aimed to investigate the role of FAF1 in IDD. Initially, the expression of FAF1 and its correlation with Pfirrmann scores were detected in patients with IDD. Subsequently, the role of FAF1 on cell growth, proliferation and apoptosis was examined in patients with IDD. The findings suggested that the overexpression of FAF1 may act as a suppressor in the development of IDD by targeting the extracellular signal-regulated (Erk) signaling pathway.

\section{Materials and methods}

Patients and tissue samples. The human central NP specimens were collected from patients with IDD ( $n=10$; average age $43.79 \pm 6.33$, range $35-61$ years), bulging ( $n=10$; average age $46.47 \pm 5.41$, range $30-62$ years), hernia $(n=10$; average age $44.82 \pm 3.95$, range 37-66 years), and spinal cord injury $(n=10$; average age $41.63 \pm 2.72$, range 31-59 years) between May 2013 and June 2016 from the Department of Orthopedics and Traumatic Surgery, Nantong Municipal Hospital of Traditional Chinese Medicine (Nantong, China). Each group comprised 5 males and 5 females. The degree of disc degeneration was determined by two spinal surgeons from a magnetic resonance imaging (MRI) scan according to the modified Pfirrmann grading classification (19). The samples graded as 1 were classified as a normal control, whereas samples graded as 3, 4 and 5 were included in bulging, hernia and IDD groups, respectively. The specimens were first isolated from the annulus fibrosus and then divided into two, which were frozen in liquid nitrogen at $-80^{\circ} \mathrm{C}$ or fixed in $4 \%$ paraformaldehyde, respectively. All samples were obtained following the provision of informed consent. The study was approved by Nantong Municipal Hospital of Traditional Chinese Medicine.

RNA Extraction and lncRNA expression assay. The NP tissues were isolated from 10 IDD and 10 spinal cord injury samples, and total RNA was extracted separately using TRIzol reagent (Invitrogen; Thermo Fisher Scientific, Inc., Waltham, MA, USA) according to the manufacturer's protocol. Following removal of the contaminating genomic DNA, the quality and quantity of the RNA were detected using a NanoDrop ND-1000 spectrophotometer (NanoDrop Technologies; Thermo Fisher Scientific, Inc., Wilmington, DE, USA) and an Agilent 2100 Bioanalyzer (Agilent Technologies, Inc., Santa Clara, CA, USA). Subsequently, gel electrophoresis was performed to measure RNA integrity. The total RNAs obtained were divided equally into IDD and normal control groups. Reverse transcription was then performed using a Reverse Transcription kit (Thermo Fisher Scientific, Inc.). The quantitative polymerase chain reaction (qPCR) analysis was performed using an Applied Biosystems 7900 Real-time PCR system (Applied Biosystems; Thermo Fisher Scientific, Inc.) using $5 \mu \mathrm{g}$ RNA (miRNA cDNA Synthesis kit; Takara Biotechnology Co., Ltd., Dalian, China), with a reaction volume of $20 \mu \mathrm{l}$. Reaction reagents $(20 \mu \mathrm{l})$ were: $10 \mu \mathrm{l} \mathrm{SYBR}$ Premix Ex Taq II, $0.8 \mu 1$ PCR Forward Primer, $0.8 \mu 1$ PCR Reverse Primer, $0.4 \mu 1$ ROX Reference Dye, $2.0 \mu 1 \mathrm{cDNA}$

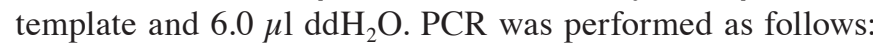
$10 \mathrm{sec}$ at $95^{\circ} \mathrm{C}, 95 \mathrm{sec}$ at $5^{\circ} \mathrm{C}$, and $34 \mathrm{sec}$ at $60^{\circ} \mathrm{C}$ for 45 cycles.
Glyceraldehyde 3-phosphate dehydrogenase (GAPDH) was used for normalization and the relative expression of FAF1was calculated using the $2^{-\Delta \Delta \mathrm{Cq}}$ method (20). The PCR primers were as follows: FAF1: Forward, AGAGCAAAGAGGGAAG, reverse, AAGAACTCGCCACTG; GAPDH: Forward, GGG AAACTGTGGCGTG AT; Reverse, GAGTGGGTGTCGCTG TTGA.

Culture of NP cells. The NP tissues were cut into sections using ophthalmic scissors, and digested with PBS containing $0.025 \%$ type II collagenase (Invitrogen; Thermo Fisher Scientific, Inc.) for $4 \mathrm{~h}$ at $37^{\circ} \mathrm{C}$. Following filtration at $200 \mu \mathrm{m}$ pore, the well-digested NP tissues were centrifuged at $500 \mathrm{x} \mathrm{g}$ for $10 \mathrm{~min}$ at room temperature and the supernatant was aspirated. The NP cells were seeded $\left(5 \times 10^{5}\right.$ cells/well $)$ into a culture flask in DMEM (Gibco; Thermo Fisher Scientific, Inc.) with $10 \%$ fetal bovine serum (FBS; Gibco; Thermo Fisher Scientific, Inc.) and $1 \%$ streptomycin/penicillin, and then placed into an incubator with $5 \% \mathrm{CO}_{2}$, saturated humidity at $37^{\circ} \mathrm{C}$ for 3 days.

Plasmids and cell transfection. The full-length sequence of FAF1 was PCR-amplified using PrimeSTAR HS DNA Polymerase (Takara Biotechnology Co., Ltd.) and cloned into the PcDNA3.1 vector (Invitrogen; Thermo Fisher Scientific, Inc.). PCR was performed as follows: $15 \mathrm{sec}$ at $95^{\circ} \mathrm{C}, 95 \mathrm{sec}$ at $5^{\circ} \mathrm{C}$, and $60 \mathrm{sec}$ at $60^{\circ} \mathrm{C}$ for 30 cycles. Short hairpin (sh) RNA-FAF1 (5'-GGCAGCGGTCCCCGAGGAGGCGGC AG-3') and shRNA-control (5'-AAGTCCCTGGTCTCT GGAGACTGTTCTG-3') were synthesized by GenePharma Co., Ltd. (Shanghai, China). The day prior to transfection, $6 \times 10^{4}$ cells were inoculated into 6-well dishes with $2 \mathrm{ml}$ medium in every well. Transfection was performed when the cell density reached 50-60\%. The diluted transfections were mixed carefully with Lipofectamine ${ }^{\circledR} 2000$ (Invitrogen; Thermo Fisher Scientific, Inc.) and cultured for $20 \mathrm{~min}$ at room temperature. The mixture of plasmid oligonucleotide and transfection reagents was added to each well containing the cells and culture medium. The cells were incubated under conditions of $37^{\circ} \mathrm{C}$ and $5 \% \mathrm{CO}_{2}$, and then collected following 48-72 h of transfection.

Cell proliferation assay. A Cell Counting Kit-8 (CCK-8; Dojindo Molecular Technologies, Inc., Kumamoto, Japan) was used to determine the proliferation of the transfected NP cells. Firstly, the cells were seeded onto 96-well plates (5x $10^{3}$ cells/well) and $10 \mu \mathrm{l} \mathrm{CCK-8} \mathrm{was} \mathrm{added} \mathrm{to} \mathrm{each} \mathrm{well} \mathrm{at}$ the indicated time (1, 2, 3 and 4 days) in accordance with the manufacturer's protocol. The cells were incubated at $37^{\circ} \mathrm{C}$ for $2 \mathrm{~h}$. According to the absorbance of each well at $450 \mathrm{~nm}$ for each group, the cell viability was assessed using a Benchmark microplate reader (Bio-Rad Laboratories, Inc., Hercules, CA, USA).

Immunofluorescence staining. Following rinsing with phosphate-buffered saline three times, the cultured NP cells were fixed with $4 \%$ formaldehyde for $10 \mathrm{~min}$, and blocked with 1\% BSA (Sigma-Aldrich; Merck KGaA, Darmstadt, Germany) for $45 \mathrm{~min}$. The cells were incubated for $1 \mathrm{~h}$ at room temperature with primary antibody against Ki67 (rabbit polyclonal antibody; ab15580; 1:200; Abcam, Cambridge, 

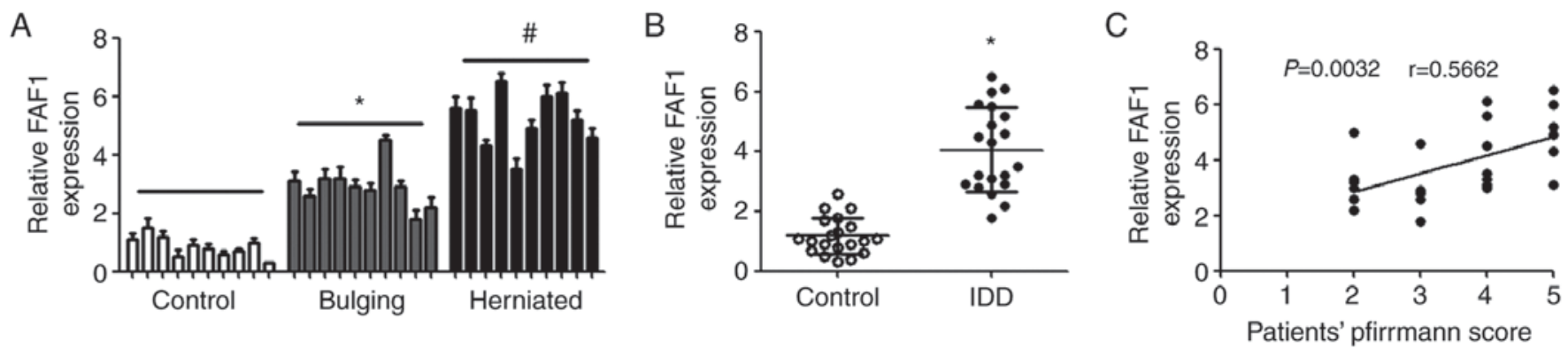

Figure 1. Differential expression of FAF1 in patients with IDD. (A) Relative expression levels of FAF1 in NP tissues from patients in the control group, bulging group and herniated group. (B) Relative expression of FAF1 in NP tissue from patients with IDD and normal controls. (C) Correlation between the expression of FAF1 and patients' Pfirrmann scores. Data are expressed as the mean \pm standard deviation. ${ }^{*} \mathrm{P}<0.05$, vs. control group; ${ }^{\sharp} \mathrm{P}<0.05$, vs. bulging group ( $\mathrm{n}=10$ ). FAF1, Fas-associated protein factor-1; IDD, intervertebral disc degeneration; NP, nucleus pulposus.

UK). The cells were then rinsed with PBS and incubated with fluorescein isothiocyanate (FITC)-conjugated goat anti-rabbit IgG antibody (goat polyclonal antibody; ab6662; 1:100; Abcam) for $1 \mathrm{~h}$ at room temperature. Subsequently, the cells were analyzed using a fluorescence microscope (Olympus, Tokyo, Japan).

Analysis of cell cycle and apoptosis. The effects of FAF1 on cell cycle and apoptosis were measured using flow cytometry. First, the cells were cultured for $48 \mathrm{~h}$, trypsinized and fixed with $70 \%$ ethanol at $-20^{\circ} \mathrm{C}$ for $12 \mathrm{~h}$ according to the manufacturer's protocol. Following washing with PBS, the cells were treated with $20 \mu \mathrm{g} / \mathrm{ml}$ RNaseA (Sigma-Aldrich; Merck $\mathrm{KGaA}$ ) for $2 \mathrm{~h}$ at $37^{\circ} \mathrm{C}$, then incubated with $50 \mu \mathrm{g} / \mathrm{ml}$ propidium iodide (Sigma-Aldrich; Merck KGaA) for $30 \mathrm{~min}$. An Annexin-V FITC Apoptosis kit (Tiangen Biotech Co., Ltd., Beijing, China) was used to assess cell apoptosis according to the manufacturer's protocol. Cell cycle and apoptosis were analyzed using FACSDiva 6.1.1 (BD Biosciences, Franklin Lakes, NJ, USA) and ModFit LT 3.0 (Verity Software House, Inc., Topsham, ME, USA) software.

Western blot analysis. Total protein was extracted from the transfected NP cells using radioimmunoprecipitation assay lysis buffer (Sigma-Aldrich; Merck KGaA) at $4^{\circ} \mathrm{C}$ for $30 \mathrm{~min}$. Then, the proteins were extracted by RIPA buffer (Sigma-Aldrich; Merck KGaA) and separated on a $10 \%$ sodium dodecyl sulphate-polyacrylamide electrophoresis gel by electrophoresis for $120 \mathrm{~min}$ and transferred onto $0.45-\mathrm{mM}$ PVDF membranes (EMD Millipore, Billerica, MA, USA). The concentration of protein was quantified by a BCA kit (Beyotime Institute of Biotechnology, Nantong, China). Following blocking in sealing fluid for $60 \mathrm{~min}$, the membranes were incubated with anti-phosphorylated (p)-Erk (rabbit polyclonal antibody; 4370; 1:1,000; Cell Signaling Technology,Inc., Danvers, MA, USA) antibodies overnight at $4^{\circ} \mathrm{C}$. Following rinsing with TBST three times, the membranes were incubated with a secondary antibody (7074; 1:1,000; anti-rabbit polyclonal antibody; Cell Signaling Technology, Inc.) for $1 \mathrm{~h}$ at room temperature. Enhanced chemiluminescence reagent (Nanjing KeyGen Biotech, Inc., Nanjing, China) was used to develop the immunoreactivity, and the protein bands were analyzed using Quantity One software version 4.62 (Bio-Rad Laboratories, Inc.). GAPDH (mouse monoclonal antibody;
97166; 1:2,000; Cell Signaling Technology, Inc.) was used for normalization.

Statistical analysis. All the data were analyzed using SPSS software version 18.0 (SPSS, Inc., Chicago, IL, USA) and expressed as the mean \pm standard deviation. Differences between two groups were evaluated using Student's t-test, and differences among three or more groups were evaluated using one-way analysis of variance. The correlation between the expression of FAF1 and Pfirrmann grades was determined using Spearman's correlation analysis. $\mathrm{P}<0.05$ was considered to indicate a statistically significant difference.

\section{Results}

Expression of FAF1 is downregulated in patients with IDD. RT-qPCR analysis was performed to detect the differential expression of FAF1 in patients with spinal cord injury, bulging, herniated intervertebral disc and IDD. Compared with the control group, the expression of FAF1 was upregulated in the patients with bulging and herniation, and the level of FAF1 in those with hernia was higher, compared with that in patients with bulging (Fig. 1A). In addition, it was found that the expression of FAF1 in patients with IDD was significantly increased, compared with that in patients with spinal cord injury (Fig. 1B). The correlations between the expression of FAF1 and the severity of IDD were also examined. The results showed that there were significant positive correlations between the expression of FAF1 and patients' Pfirrmann scores (Fig. 1C).

Overexpression of FAF1 promotes NP cell proliferation. To investigate the role of FAF1 in NP cells, the FAF1 expression vector and FAF1-specific shRNA were transfected into NP cells (Fig. 2A). The effect of FAF1 on NP cell proliferation was measured using a CCK-8 assay. The overexpression of FAF1 in NP cells significantly promoted cell growth, compared with that in the control group $(\mathrm{P}<0.05)$; the shRNA-FAF1 group exhibited decreased cell growth, compared with that in the shRNA-ctrl group (Fig. 2B). The results of the immunofluorescence staining showed similar results; Ki67 fluorescence intensity was upregulated in the FAF1-overexpressing group and downregulated in the shRNA-FAF1 group, compared with intensity in the corresponding control groups (Fig. 2C and D). 

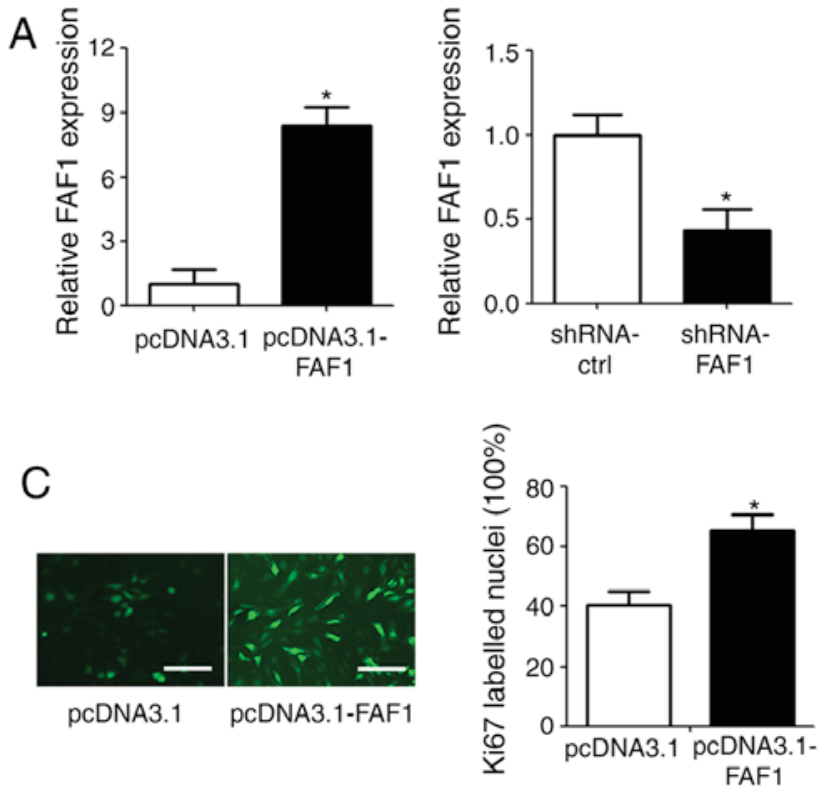
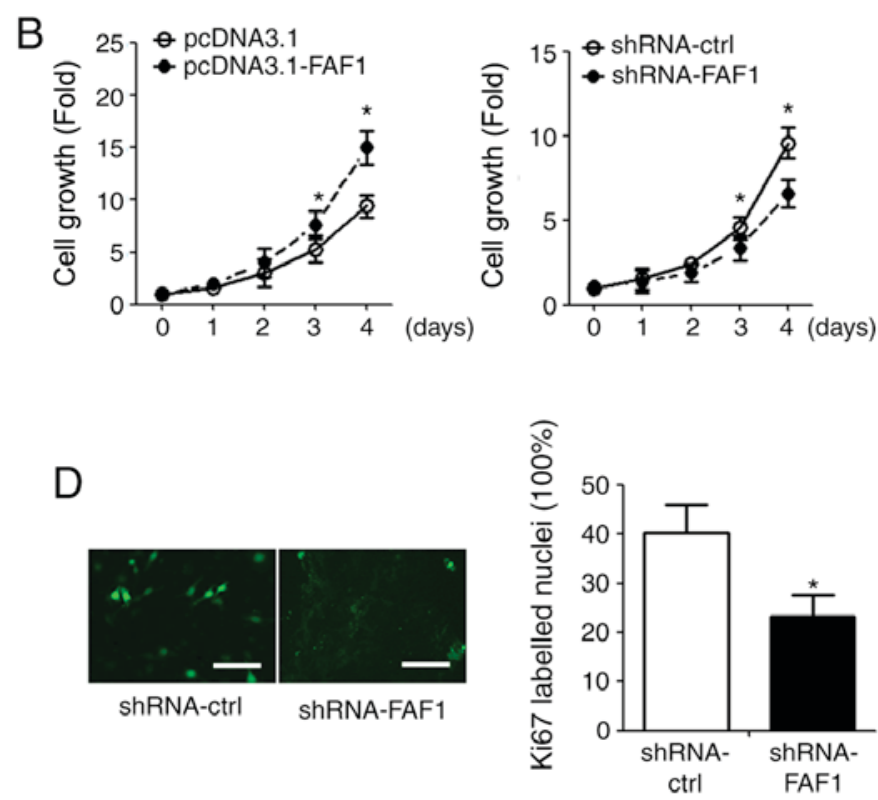

Figure 2. FAF1 exerts an essential function in NP cell proliferation. The NP cells were transfected with FAF1 expression vector, shRNA and control for $48 \mathrm{~h}$. (A) Expression levels of FAF1 were detected using reverse transcription-quantitative polymerase chain reaction analysis following transfection. (B) A CCK-8 assay was performed to examine the proliferation of NP cells at the indicated time points. Immunofluorescence staining showed the expression of Ki67 in NP cells following transfection in the (C) FAF-overexpression and (D) FAF-knockdown groups (scale bar=100 $\mu \mathrm{m}$ ). Data are expressed as the mean \pm standard deviation. "P<0.05, vs. control group (n=5). 10. FAF1, Fas-associated protein factor-1; NP, nucleus pulposus; shRNA, short hairpin RNA; ctrl, control.
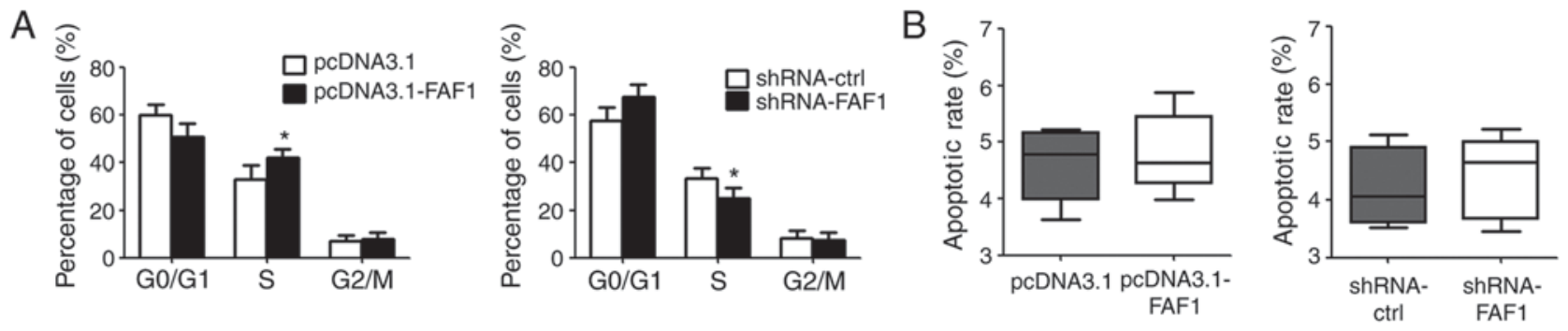

Figure 3. Effects of FAF1 on cell cycle and apoptosis. The NP cells were transfected with the FAF1 expression vector, shRNA and control for 48 h. (A) Flow cytometry determined the percentage of cells in each phase of the cell cycle in NP cells. (B) Apoptotic rates were analyzed using flow cytometry. Data are expressed as the mean \pm standard deviation. ${ }^{*} \mathrm{P}<0.05$, vs. control group $(\mathrm{n}=5)$. FAF1, Fas-associated protein factor-1; NP, nucleus pulposus; shRNA, short hairpin RNA; ctrl, control.

FAF1 increases the percentage of cells in the S-phase of the cell cycle. To elucidate the pro-growth mechanism of FAF1 in NP cells, the present study assessed the cell cycle and cell apoptosis. According to the results of the flow cytometric assay, the overexpression of FAF1 in NP cells increased the percentage of cells in the S-phase, compared with that in the control group. By contrast, the downregulation of FAF1 in NP cells reduced the percentage of cells in the S-phase (Fig. 3A). However, no significant differences in apoptotic rate were found between the FAF1-overexpressing group or shRNA-FAF1 group and their corresponding control groups (Fig. 3B).

FAF1 promotes NP cell proliferation directly via targeting the Erk signaling pathway. The results of the western blot analysis showed that the expression of p-Erk increased with the presence of the FAF1 expression vector. By contrast, the level of p-Erk in the shRNA-FAF1 group was decreased, compared with that in the control group (Fig. 4A). To investigate whether
FAF1 promoted NP cell proliferation by modulating Erk signaling pathway activation, PD98059 was used to inhibit the activation of the Erk signaling pathway. The results showed that the expression of p-Erk decreased sharply following treatment with PD98059 (Fig. 4B). The results showed that PD98059 eliminated the FAF1-induced cell proliferation in NP cells (Fig. 4C). Similarly, the Ki67 immunofluorescence staining and flow cytometry indicated that PD98059 reduced the percentage of Ki67-labelled nuclei and S-phase cells in the FAF1-overexpressing NP cells (Fig. 4D and E).

\section{Discussion}

Patients with IDD suffer from pain physically and psychologically. The investigation of novel diagnostic and therapeutic strategies is required to assist in improving patient quality of life $(6,21-23)$. Accumulating evidence has demonstrated that IncRNAs are important in the development and progression of IDD $(24,25)$. At present, the diagnosis of IDD is based on 
A
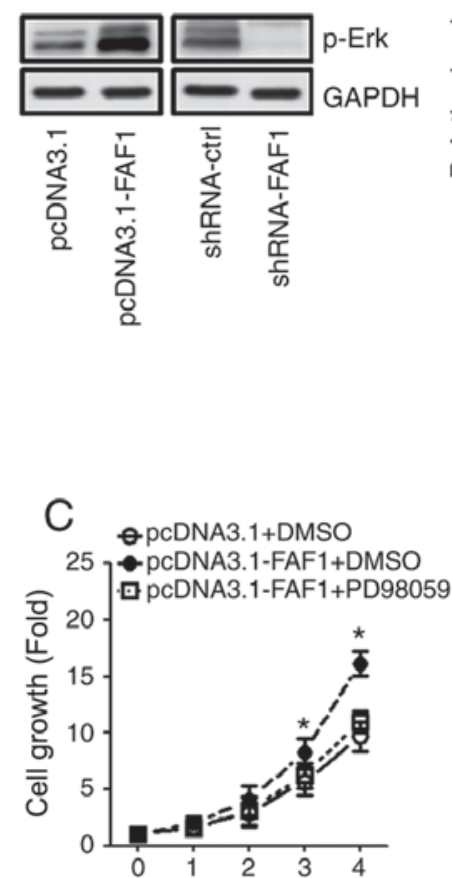

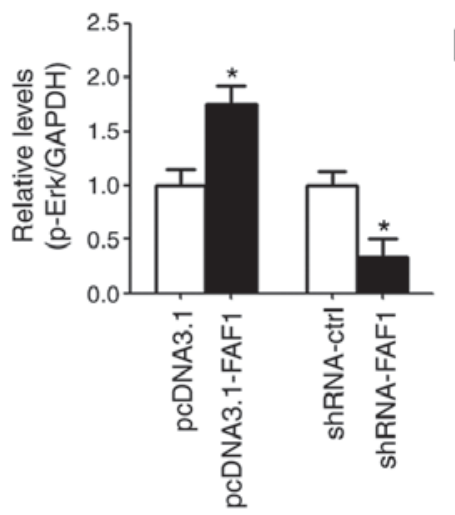

D

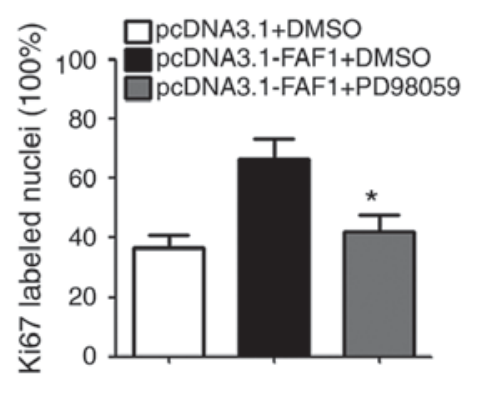

B

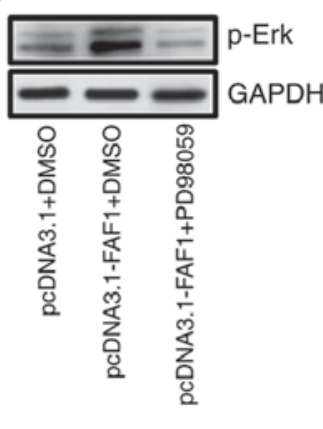

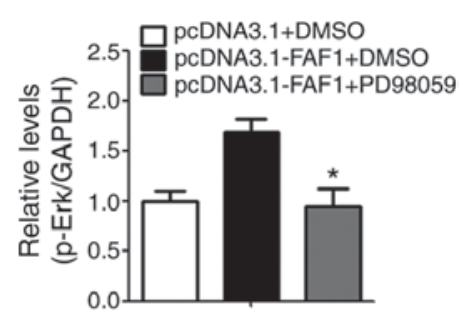

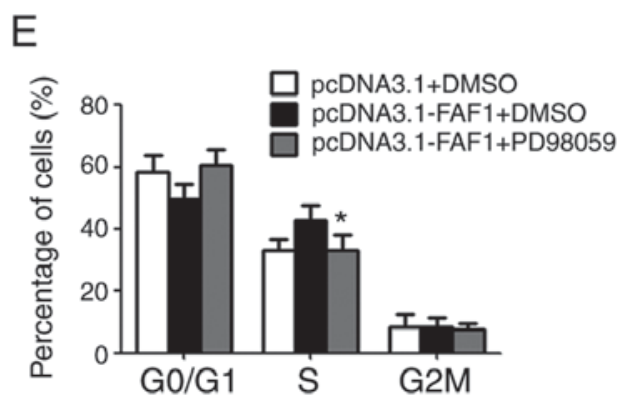

Figure 4. FAF1 regulates NP cell proliferation via controlling the Erk signaling pathway. The NP cells were transfected with the FAF1 expression vector, shRNA and control for $48 \mathrm{~h}$. For inactivation of the Erk signaling pathway, NP cells were treated with the inhibitor, PD98059 (10 $\mu \mathrm{M}$ ). (A and B) Expression levels of p-Erk were measured using western blot analysis. (C) NP cell growth was detected using a Cell Counting Kit-8 assay. (D) NP cell proliferation was evaluated using Ki67 immunofluorescence staining. (E) Cell cycle assays. Data are expressed as the mean \pm standard deviation. ${ }^{*} \mathrm{P}<0.05$, vs. PcDNA3.1-FAF1+DMSO group ( $\mathrm{n}=5$ ). FAF1, Fas-associated protein factor-1; NP, nucleus pulposus; Erk, extracellular signal-regulated kinase; p-Erk, phosphorylated Erk; shRNA, short hairpin RNA.

advanced imaging techniques, including MRI (26). However, the information provided by MRI reveals only intermediate or late stages of IDD, whereas the degradation of proteoglycans, particularly aggrecan, and extracellular matrix changes are early signs of IDD. Therefore, the investigation of more precise diagnostic methods for the initial stage of IDD is required. It has been reported that certain small molecules, including lncRNAs, are associated with IDD, and that the dysregulation of these molecules is key in the progression of gene transcription (27).

In the present study, the expression of FAF1 was analyzed in 40 tissue samples from patients with IDD, bulging, herniation and spinal cord injury. The results demonstrated that the expression level of FAF1 was upregulated, with the degree of aggravated degeneration and the expression of FAF1 in IDD being significantly higher, compared with those of the control group, suggesting that FAF1 may be closely associated with the development of IDD. In addition, patients' Pfirrmann scores showed that the expression of FAF1 was positively correlated with the grade of disc degeneration, which suggested that FAF1 may act as a promoter in the development of IDD. To confirm the role of FAF1, FAF was overexpressed or knocked down in primary NP cells. The CCK-8 assay and immunofluorescence staining demonstrated that the overexpression of FAF1 promoted NP cell proliferation and that the knockdown of FAF1 suppressed NP cell proliferation. Consistently, a previous study reported that FAF1 was upregulated in degenerative discs (2). The present study also found that FAF1 regulated NP cell proliferation via controlling cell cycle.
These results indicated that FAF1 may be a novel inducer in the progression of IDD by promoting cell proliferation.

To elucidate the molecular mechanism underlying the effect of FAF1, p-Erk was confirmed as a target gene of FAF1, and it was found that the expression of FAF1 was positively associated with p-Erk. Erk is one of the members of the mitogen-activated protein kinase pathway, which is important in cell survival, cell growth and programmed cell death signaling events (28). p-Erk is reported to be closely associated with cell survival and cell growth in several human diseases, including ovarian cancer (29) and hepatotoxicity (30). The present study showed that p-Erk was upregulated with the overexpression of FAF1, but downregulated when FAF1 was knocked down, compared with that in the control group, suggesting that FAF1 may be connected with Erk signaling pathway activation. In addition, PD98059, an inhibitor of the Erk signaling pathway, eliminated FAF1-induced cell proliferation in NP cells. These results indicated that FAF1 may promote cell proliferation by modulating Erk pathway activation, which is consistent with the results of previous studies $(29,30)$.

In conclusion, the present study found that the expression of FAF1 was upregulated in IDD and that the expression level of FAF1 was positively correlated with the grade of disc degeneration. These results elucidated an important molecular mechanism, in which FAF1 exerted a positive effect on cell proliferation in IDD by upregulating p-Erk. Taken together, FAF1 may be a novel marker in the early diagnosis of IDD and a therapeutic target for patients. 


\section{References}

1. Vasiliadis ES, Pneumaticos SG, Evangelopoulos DS and Papavassiliou AG: Biologic treatment of mild and moderate intervertebral disc degeneration. Mol Med 20: 400-409, 2014.

2. Liu H, Huang X, Liu X, Xiao S, Zhang Y, Xiang T, Shen X, Wang $\mathrm{G}$ and Sheng B: miR-21 promotes human nucleus pulposus cell proliferation through PTEN/AKT signaling. Int J Mol Sci 15 4007-4018, 2014.

3. Jarman JP, Arpinar VE, Baruah D, Klein AP, Maiman DJ and Muftuler LT: Intervertebral disc height loss demonstrates the threshold of major pathological changes during degeneration. Eur Spine J 24: 1944-1950, 2015.

4. Samartzis D, Karppinen J, Mok F, Fong DY, Luk KD and Cheung KM: A population-based study of juvenile disc degeneration and its association with overweight and obesity, low back pain and diminished functional status. J Bone Joint Surg Am 93: 662-670, 2011.

5. Bolesta MJ: Commentary on an article by Dino Samartzis, DSc, et al.: 'A population-based study of juvenile disc degeneration and its association with overweight and obesity, low back pain and diminished functional status'. J Bone Joint Surg Am 93: e34, 2011.

6. Freemont AJ: The cellular pathobiology of the degenerate intervertebral disc and discogenic back pain. Rheumatology (Oxford) 48: 5-10, 2009.

7. Li Z, Yu X, Shen J, Chan MT and Wu WK: MicroRNA in intervertebral disc degeneration. Cell Prolif 48: 278-283, 2015.

8. Zhao B, Yu Q, Li H, Guo X and He X: Characterization of microRNA expression profiles in patients with intervertebral disc degeneration. Int J Mol Med 33: 43-50, 2014.

9. Hu P, Feng B, Wang G, Ning B and Jia T: Microarray based analysis of gene regulation by microRNA in intervertebral disc degeneration. Mol Med Rep 12: 4925-4930, 2015.

10. Fatica A and Bozzoni I: Long non-coding RNAs: New players in cell differentiation and development. Nat Rev Genet 15: 7-21, 2014.

11. Mercer TR and Mattick JS: Structure and function of long noncoding RNAs in epigenetic regulation. Nat Struct Mol Biol 20: 300-307, 2013.

12. Lee JT: Epigenetic regulation by long noncoding RNAs. Science 338: 1435-1439, 2012.

13. Mang Y, Li L, Ran J, Zhang S, Liu J, Li L, Chen Y, Liu J, Gao Y and Ren G: Long noncoding RNA NEAT1 promotes cell proliferation and invasion by regulating hnRNP A2 expression in hepatocellular carcinoma cells. Onco Targets Ther 10: 1003-1016, 2017.

14. Chen T, Wang H, Yang P and He ZY: Prognostic role of long noncoding RNA NEAT1 in various carcinomas: A meta-analysis. Onco Targets Ther 10: 993-1000, 2017.

15. Zhang LQ, Yang SQ, Wang Y, Fang Q, Chen XJ, Lu HS and Zhao LP: Long noncoding RNA MIR4697HG promotes cell growth and metastasis in human ovarian cancer. Anal Cell Pathol (Amst) 2017: 8267863, 2017.

16. Sookoian S, Rohr C, Salatino A, Dopazo H, Fernandez Gianotti T, Castaño GO and Pirola CJ: Genetic variation in long noncoding RNAs and the risk of nonalcoholic fatty liver disease. Oncotarget 8: 22917-22926, 2017.
17. Song J, Park JK, Lee JJ, Choi YS, Ryu KS, Kim JH, Kim E, Lee KJ, Jeon YH and Kim EE: Structure and interaction of ubiquitin-associated domain of human Fas-associated factor 1. Protein Sci 18: 2265-2276, 2009.

18. Wan ZY, Song F, Sun Z, Chen YF, Zhang WL, Samartzis D, Ma CJ, Che L, Liu X, Ali MA, et al: Aberrantly expressed long noncoding RNAs in human intervertebral disc degeneration: A microarray related study. Arthritis Res Ther 16: 465, 2014.

19. Urrutia J, Besa P, Campos M, Cikutovic P, Cabezon M, Molina M and Cruz JP: The Pfirrmann classification of lumbar intervertebral disc degeneration: An independent inter- and intra-observer agreement assessment. Eur Spine J 25: 2728-2733, 2016.

20. Livak KJ and Schmittgen TD: Analysis of relative gene expression data using real-time quantitative PCR and the 2(-Delta Delta C(T)) method. Methods 25: 402-408, 2001.

21. Gore M, Sadosky A, Stacey BR, Tai KS and Leslie D: The burden of chronic low back pain: Clinical comorbidities, treatment patterns and health care costs in usual care settings. Spine (Phila Pa 1976) 37: E668-E677, 2012.

22. Katz JN: Lumbar disc disorders and low-back pain: Socioeconomic factors and consequences. J Bone Joint Surg Am 2 (88 Suppl): S21-S24, 2006.

23. Parthan A, Evans CJ and Le K: Chronic low back pain: Epidemiology, economic burden and patient-reported outcomes in the USA. Expert Rev Pharmacoecon Outcomes Res 6: 359-369, 2006.

24. Zhao B, Lu M, Wang D, Li H and He X: Genome-wide identification of long noncoding RNAs in human intervertebral disc degeneration by RNA sequencing. Biomed Res Int 2016: 3684875,2016

25. Chen Y, Ni H, Zhao Y, Chen K, Li M, Li C, Zhu X and Fu Q: Potential role of lncRNAs in contributing to pathogenesis of intervertebral disc degeneration based on microarray data. Med Sci Monit 21: 3449-3458, 2015.

26. Mehra M, Hill K, Nicholl D and Schadrack J: The burden of chronic low back pain with and without a neuropathic component: A healthcare resource use and cost analysis. J Med Econ 15: 245-252, 2012

27. Ohrt-Nissen S, Døssing KB, Rossing M, Lajer C, Vikeså J, Nielsen FC, Friis-Hansen L and Dahl B: Characterization of miRNA expression in human degenerative lumbar discs. Connect Tissue Res 54: 197-203, 2013.

28. Zheng J, Son DJ, Lee HL, Lee HP, Kim TH, Joo JH, Ham YW, Kim WJ, Jung JK, Han SB and Hong JT: (E)-2-methoxy4-(3-(4-methoxyphenyl)prop-1-en-1-yl)phenol suppresses ovarian cancer cell growth via inhibition of ERK and STAT3. Mol Carcinog 56: 2003-2013, 2017.

29. Kandala PK, Wright SE and Srivastava SK: Blocking epidermal growth factor receptor activation by 3,3'-diindolylmethane suppresses ovarian tumor growth in vitro and in vivo. J Pharmacol Exp Ther 341: 24-32, 2012.

30. Gao XP, Qian DW, Xie Z and Hui H: Protective role of licochalcone B against ethanol-induced hepatotoxicity through regulation of Erk signaling. Iran J Basic Med Sci 20: 131-137, 2017. 